\title{
Comparison of the expression of complement regulatory proteins CD46, CD55 and CD59 in primary colon cancer and synchronous/ metachronous liver metastases
}

\author{
EWA WILCZEKI , ALEKSANDER WASIUTYNSKII, GRZEGORZ M. WILCZYNSKI ${ }^{2}$, \\ DARIUSZ SLADOWSKI ${ }^{3}$ BARBARA GORNICKA ${ }^{l}$ \\ ${ }^{1}$ Department of Pathology, Medical University of Warsaw, Warsaw, Poland \\ ${ }^{2}$ Laboratory of Molecular and Systemic Neuromorphology, Nencki Institute of Experimental Biology, Warsaw, Poland \\ ${ }^{3}$ Department of Transplantology and Central Tissue Bank, Centre for Biostructure, Medical University of Warsaw, Warsaw, Poland
}

\begin{abstract}
Although recent years have brought many advantages in clinical oncology, colon cancer is still a serious problem. Surgical resection remains the most efficient treatment, but its results are not always satisfactory. To improve disease-free survival, overall survival, and to prevent metastases, new strategies for effective treatment are searched for. Immunotherapy using monoclonal antibodies was shown to be effective in some types of malignancies, however one of the most important obstacles to this type of treatment is attributed to the presence of the complement inhibitors on cancer cells. The three main membrane-bound complement inhibitors that limit the efficacy of the complement-mediated cytotoxicity on cancer cells are CD46, CD55 and CD59. In the current study, we evaluated the expression of these three complement inhibitors on colon cancer tumor samples. Additionally we compared, in samples obtained from the same patients, the immunoreactivities of these inhibitors in the primary and metastatic sites of the tumor. We found that in colon cancer cells the most common is CD46, whereas CD55 and CD59 are present in a small percentage of tumor samples. Although the staining intensity varied between primary and metastatic foci, these results, regarding all examined complement inhibitors were not statistically relevant. It appears that CD46 plays the most significant role among complement inhibitors in colon cancer. Thus, attempts should be made to inhibit this regulator when complement-mediated cytotoxicity is taken into consideration as an option for control of cancer cells remaining after surgical resection of the tumor.
\end{abstract}

Key words: colon cancer, CD46, CD55, CD59.

(Centr Eur J Immunol 2013; 38 (4): 543-548)

\section{Introduction}

One promising approach in cancer treatment seems to be immunotherapy using monoclonal antibodies. The simplest variation of this method utilizes monoclonal antibodies directed against tumor-associated antigens, which can unleash natural anti-tumor capabilities of the immune system, including complement-dependent cytotoxicity (CDC). Many clinical trials indicate that although this therapy is devoid of serious side effects, it has most often insufficient efficacy. So far, the most successful immunotherapy is the treatment of B-cell lymphoma with an antibody rituximab, which recognizes and binds to the CD20 molecule. In half of the patients, treatment with this antibody induces CDC, and some of them have complete remission [1].
Numerous literature data suggest that one of the main factors, which limits effectiveness of immunotherapy is the presence of complement inhibitors on cancer cells, which are able to inactivate the complement cascade and thereby circumvent complement-mediated immune response. The complement system consists of a series of about 30 proteins, which upon activation at the cell surface, interact with one another in a cascade manner. The terminal effect of activation of the complement system is the formation of the membrane attack complex (MAC, C5b-9), whose accumulation ultimately leads to cell lysis. Activation may occur via three different pathways. In the classical pathway, the activating element is the complex antigen-antibody. Two other pathways, alternative and lectin ones, are antibody-independent. Under phys-

Correspondence: Ewa Wilczek, PhD, Department of Pathology, Medical University of Warsaw, Warsaw, Poland,

e-mail: ewa.wilczek@wum.edu.pl 
iological conditions, this system protects the host from invading microorganisms, and provides tissue homeostasis by destroying altered self (apoptotic or necrotic) cells. An excessive activation of the complement system is strictly regulated by two groups of regulatory proteins, membrane-bound (membrane complement regulatory protein, mCRP) and fluid-phase inhibitors. The most prominent among the former group of inhibitors are: CD35 (complement receptor 1, CR1), CD46 (membrane cofactor protein, MCP), CD55 (decay-accelerating factor, DAF) and CD59 (protectin). The latter two proteins are anchored to the cell membrane through a glycophosphatidylinositol (GPI) moiety, which enables motility within the membrane. It was shown that mCRP proteins are expressed by different cancer cells protecting them against complement-based destruction [2-4].

From a therapeutic point of view it is important to analyze whether the expression of complement inhibitors changes during the carcinogenesis process, what can possibly be connected with different susceptibility to the immunotherapy of cancer at different stages of progression. The aim of the study was to investigate the expression level of three main complement membrane-bound regulators in primary and metastatic colon cancer.

\section{Material and methods}

\section{Patient samples}

Tissue specimens were obtained from 40 patients undergoing surgeries because of colon cancer and liver metastasis. Tissue samples from normal colon, colon carcinoma, normal liver and liver with metastases were studied. Samples were fixed in $4 \%$ buffered formaldehyde and paraffin-embedded according to standard procedures.

\section{Immunohistochemistry}

Analysis was performed in $4 \mu \mathrm{m}$ paraffin sections. For the detection of CD55, CD46 and CD59, MCA1614, MCA2113 and MCA1024, mouse monoclonal antibodies (Serotec, Oxford, United Kingdom) were used. For the primary antibody detection, peroxidase-conjugated anti-mouse ImPress ${ }^{\mathrm{TM}}$ Reagent Kit (Vector Laboratories, Burlingame, CA) was used. To prevent endogenous biotin binding, Avidin/Biotin Blocking Kit (Vector Laboratories) was applied prior to addition of the primary antibody. Visualization of reactions was carried out using 3,3'-diaminobenzidine as a chromogen. As a control for immunoreaction specificity, tissue samples were immunostained using irrelevant mouse $\operatorname{IgG}_{1}$ (Sigma-Aldrich, St. Louis, MO) instead of the primary antibody. For analysis, 100 cells in 5 representative HPF (high power fields) were counted and immunopositive cells were presented in percentages.

\section{Results}

\section{CD46 immunoreactivity}

In the majority of biopsies (80\%), from the primary and metastatic sites, the CD46 immunoreactivity was present in epithelial cells (Table 1).

The staining pattern was predominantly membranous, with the most striking staining of lateral surfaces (Fig. 1). Apical and basal surfaces were stained less intensively. Additionally, we observed granular, cytoplasmic CD46 immunoreactivity in a small percentage of cancer cells. In about $20 \%$ of biopsies we did not find CD46 immunoreactivity. The staining intensity was mostly weak to moderate (in both groups). There was no statistically significant differences in CD46 immunoreactivity between primary and metastatic sites (Fig. 1A vs. B).

In the normal colon, CD46 was seen in the form of weak, cytoplasmic staining of epithelial cells. In some crypts, strong, granular staining was seen (Fig. 2A).

Normal hepatocytes were weakly, homogenously stained or did not show CD46 immunoreactivity. The type of staining was only cytoplasmic, no membranous reactivity was seen. Bile ducts were negative for the CD46 immunoreactivity. Negative control with irrelevant mouse immunoglobulin applied instead of the primary antibody displayed no reaction (Fig. 2B).

Table 1. The percentage of cells positive for CD46, CD55 and CD59 immunoreactivity in primary and metastatic colon cancer; the total number of counted cells was 500 in each experimental group

\begin{tabular}{|c|c|c|c|c|c|c|}
\hline $\begin{array}{l}\text { No. of } \\
\text { immun }\end{array}$ & sitive cells & $0 \%$ & $1-25 \%$ & $26-50 \%$ & $51-75 \%$ & $76-100 \%$ \\
\hline \multirow[t]{2}{*}{ CD46 } & primary tumor & 22 & 17 & 17 & 22 & 22 \\
\hline & metastasis & 18 & 13 & 13 & 17 & 39 \\
\hline \multirow[t]{2}{*}{ CD55 } & primary tumor & 38 & 38 & 10 & 4 & 10 \\
\hline & metastasis & 43 & 33 & 5 & 5 & 14 \\
\hline \multirow[t]{2}{*}{ CD59 } & primary tumor & 78 & 22 & 0 & 0 & 0 \\
\hline & metastasis & 41 & 17 & 7 & 0 & 0 \\
\hline
\end{tabular}



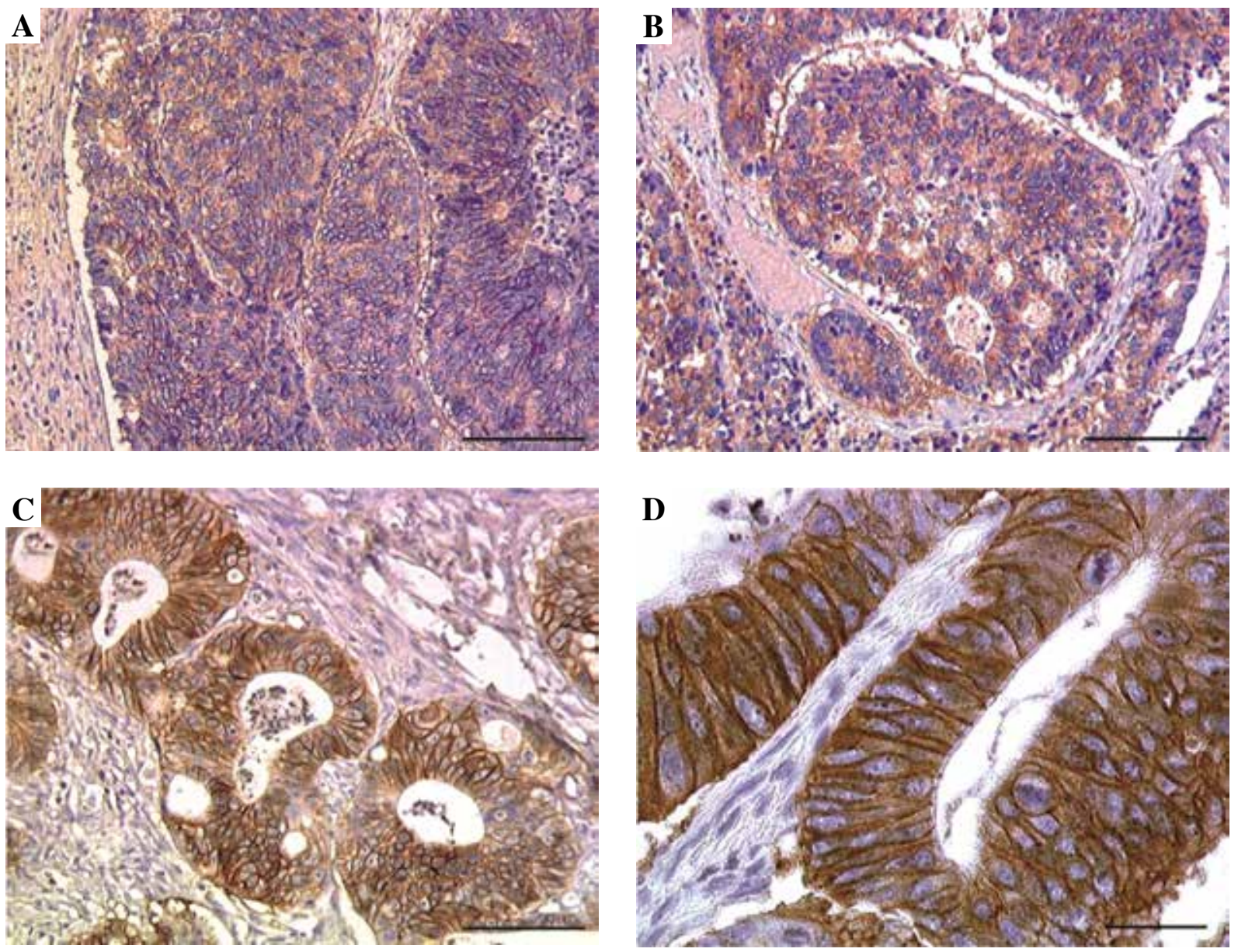

Fig. 1. Comparison of the CD46 immunoreactivity in primary (A) and metastatic (B) colon cancer, C, D an example of the strong immunostaining pattern of CD46 in the primary colon cancer section; note the most intensive staining of lateral surfaces; scale bar: A, B - $200 \mu \mathrm{m}, \mathrm{C}-100 \mu \mathrm{m}, \mathrm{D}-20 \mu \mathrm{m}$
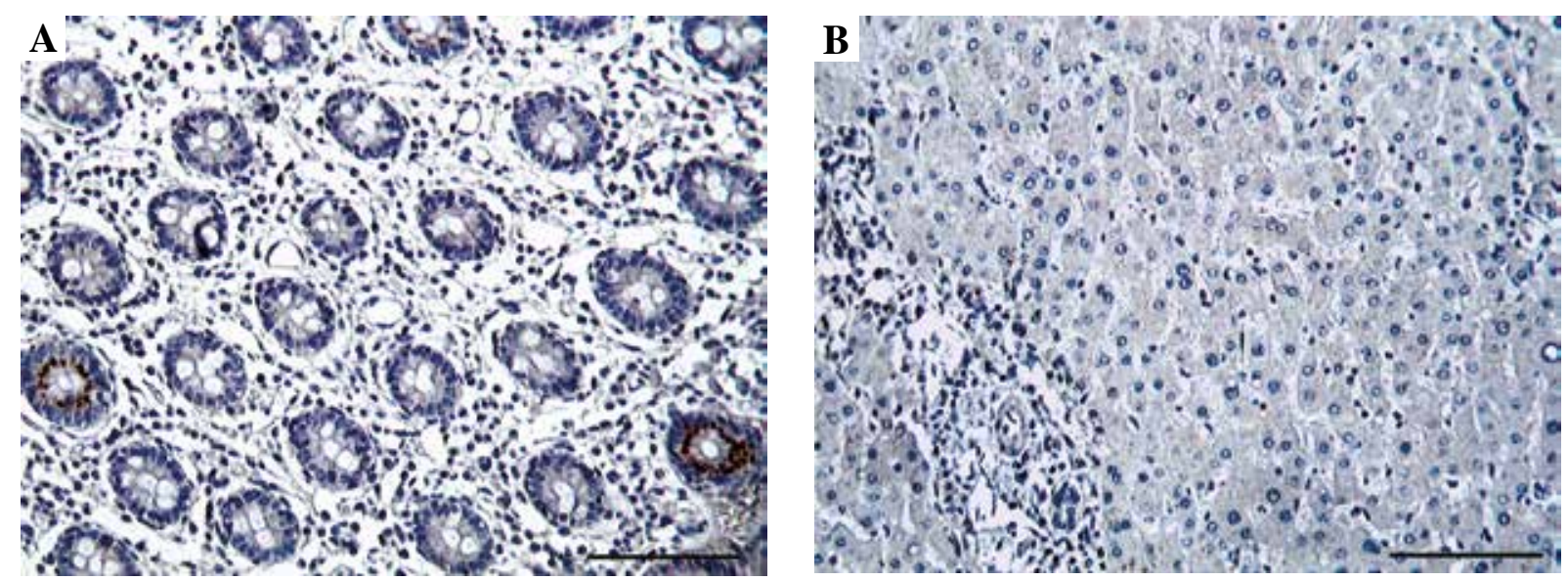

Fig. 2. CD46 immunoreactivity in the normal colon; note the selective staining in the form of large granules in individual crypts (A); a negative control, liver tissue sample stained with mouse gamma globulins instead of the primary antibody (B); scale bar: $200 \mu \mathrm{m}$ 

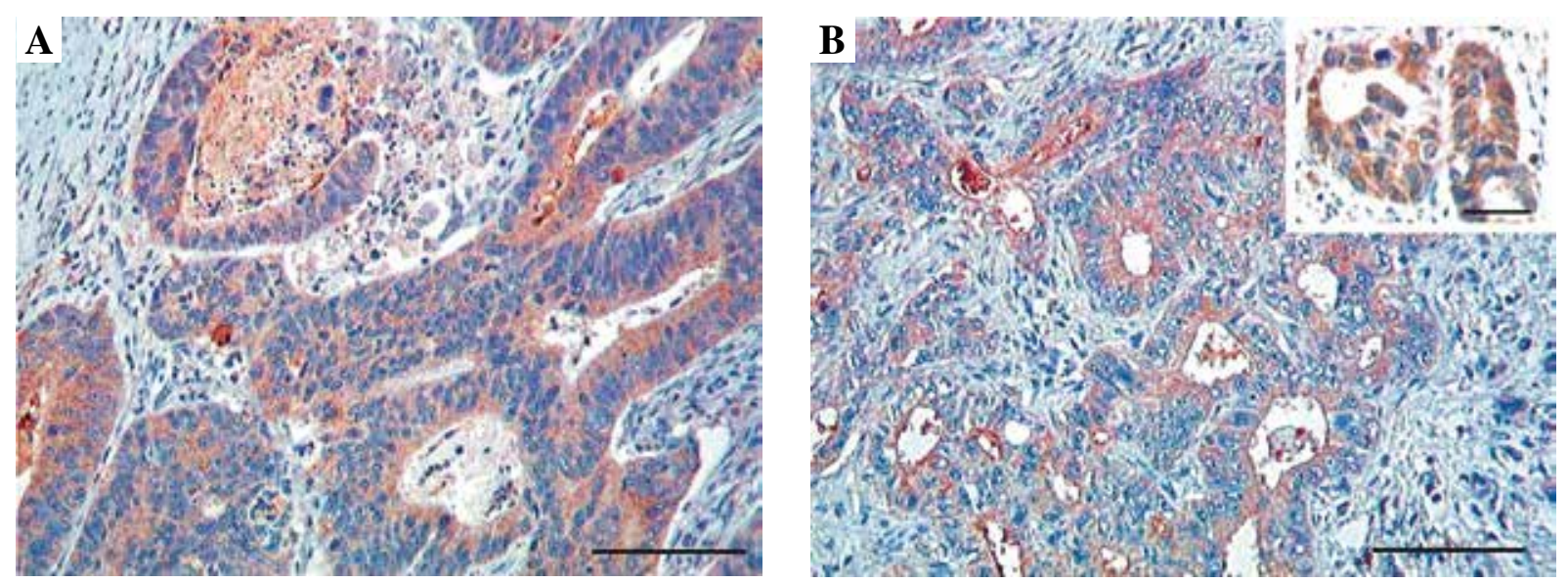

Fig. 3. Comparison of the CD55 immunoreactivity in primary (A) and metastatic (B) colon cancer; note the staining present mainly in the cytoplasm of cancer cells; scale bar: $200 \mu \mathrm{m}$, insert $100 \mu \mathrm{m}$

\section{CD55 immunoreactivity}

DAF immunoreactivity was detected in a similar percentage of primary and metastatic colon cancer (Table 1). The intensity of staining was weak in the majority of tissues. The pattern was predominantly cytoplasmic, in some biopsies apical cell surface staining of individual crypts was present (Fig. 3). In a small percentage of biopsies, we observed heterogeneous staining around small cancer tubules. The staining intensity was predominantly weak. Two mucinous colon cancer biopsies showed strong CD55 immunoreactivity localized mainly in apical and basal surfaces of cancer tubules, associated with strong staining of lateral surfaces of cancer cell membranes. Normal colon and liver biopsies were devoid of CD55 immunoreactivity.

\section{CD59 immunoreactivity}

Among all complement inhibitors examined, CD59 immunoreactivity was found in the smallest percentage of

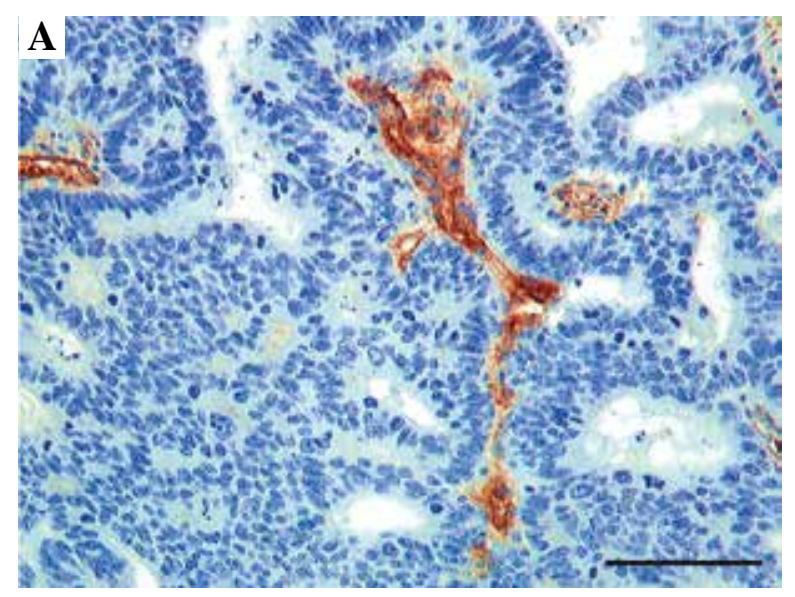

biopsies. Only $22 \%$ of primary colon cancer tissues and $24 \%$ of corresponding liver metastases showed positive staining for CD59. The intensity of staining was weak in the majority of specimens (Fig. 4). The pattern of staining was membranous, with a slightly stronger reactivity in apical surfaces of cancer tubules. In biopsies from liver metastases, very strong immunostaining was seen on residual biliary ducts. Equally strong was the immunoreactivity of the remaining hepatocytes. Very common was the presence of the CD59 immunoreactivity in endothelial cells of blood vessels, both in the normal and cancer tissue.

In the normal colon CD59 was present in individual cells of crypts and in the extracellular matrix. In the muscular layer of the mucosa, diffused CD59 immunoreactivity was present. Very strong staining was seen in submucosal nerve aggregates. In the normal liver, CD59 was mainly seen in blood vessels and bile ducts. The most intensive staining was observed in the central vein of the hepatic lobule. In some hepatocytes, weak, diffuse CD59

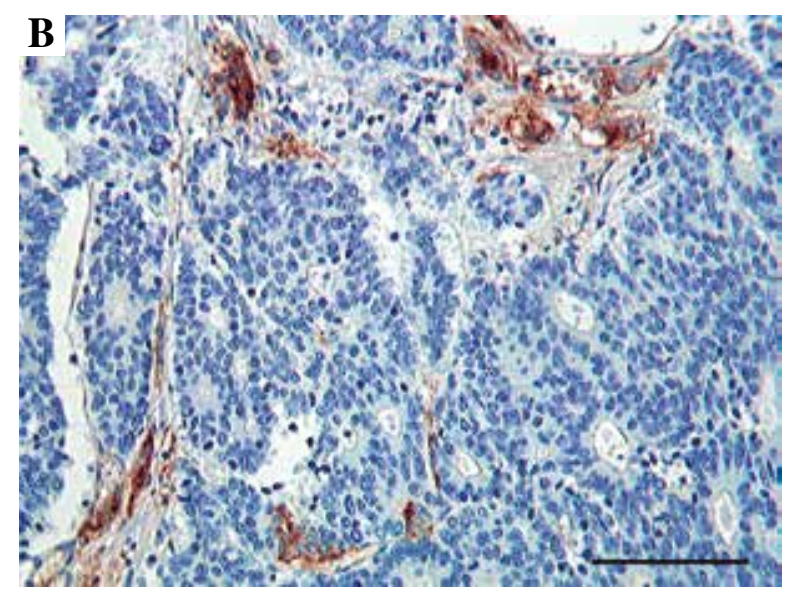

Fig. 4. Comparison of the CD59 immunoreactivity in primary (A) and metastatic (B) colon cancer; note the scarce staining of cancer cells, with the stronger immunoreactivity present in blood vessels; scale bar: $200 \mu \mathrm{m}$ 
immunoreactivity was seen, although the majority of hepatocytes were CD59-negative.

\section{Discussion}

In our work we present that among three main membrane-bound complement inhibitors, the most commonly present protein in colon cancer is CD46. CD55 and CD59 inhibitors are present to a lesser degree, in both primary and metastatic colon cancer tissue.

Despite extensive screening programs and advanced methods of treatment, the outcome of colon cancer treatment is still very unfavorable. Over half of the patients develop a recurrence in the form of liver metastases. This suggests that even successful surgery leaves in the organism tumor cells, which are difficult to be detected by the existing diagnostic procedures. The majority of surviving tumor cells are in a "sleep" mode or arrested in the cell cycle, which makes them inaccessible to standard therapy. Therefore, the search for new, effective treatments that can destroy cancer cells regardless of their status in the cell cycle is very intensive. Immunotherapy with monoclonal antibodies, although does not produce expected results in the treatment of primary colorectal cancer, may be much more effective when applied to treat micrometastases, and to control cancer cells remaining after surgical resection [5]. Attempts to combine chemotherapy and immunotherapy indicate that at least some chemotherapeutic agents enhance the efficiency of the complement system and the cell death under the influence of monoclonal antibodies, which improves the overall efficiency of the treatment [6].

As described, there is a limitation on the use of monoclonal antibodies in the treatment of solid tumors. Some authors have suggested that failure of this type of therapy is due to the inability to select those patients in whom immunotherapy is likely to be effective [7, 8]. Additionally, the key role that reduces the effectiveness of this therapy is attributed to the presence of inhibitors of the complement system on the membrane surface of tumor cells.

Results obtained in our study indicate that the overexpression of CD46 is a common phenomenon in colorectal cancer, as it concerns a high percentage of tumors. These results are consistent with a few, as of now, studies on this inhibitor. Most authors agree that CD46 expression is increased in cancer cells [9-11].

The research of CD55 presence in colon cancer cells are not consistent. CD55 expression appears to be increased in the majority of cancers and adenomas [9, 12] when compared to the normal epithelium. Only Schmitt et al. found no expression of CD55 in cancers [10]. CD55 localization was observed mainly in the apical part, usually interpreted as the peak surface of the cell membrane, of cancer cells formed in tubules $[9,12,13]$. Additionally, these authors found strong CD55 immunoreactivity localized in the tumor stroma.
Attempts have also been made to assess the prognostic value of the expression of membrane bound regulators. As Durrant et al. stated, a high expression of CD55 correlated negatively with the outcome in patients with primary colorectal cancer tumors [14]. In a group of 136 patients, the correlation of CD55 expression in tumor biopsies and 7 -year survival of patients was assessed. The study revealed that increased levels of CD55 expression (present in $24 \%$ of patients) significantly correlated with shorter survival when compared to patients with low CD55 immunoreactivity. These authors suggest CD55 as a marker of aggressiveness of colorectal cancers.

The results obtained in our study suggest that the level of CD55 expression is high only in the case of mucinous carcinomas, which is consistent with observations of other authors [15]. In the remaining group of tumors, CD55 expression, although present, showed a slightly enhanced immunoreactivity. When comparing these results with those obtained by other authors we can conclude that colorectal cancer is heterogeneous in terms of the level of CD55 expression.

Literature data on the expression and localization of the CD59 in the primary loci of colorectal cancer are diverse. Bjřrge et al. found an increased expression of CD59 in carcinomas relative to the normal epithelium, whereas according to Inoue et al., CD59 expression is increased in adenomas and reduced in carcinomas $[12,16]$. Koretz et al. highlight a different expression of CD59 in both the normal mucosa and in adenomas and carcinomas. These authors also attempted to determine the prevalence of CD59 in adenomas and metastatic carcinomas, but on a smaller number of biopsies. In metastatic tumors, however, they noted that in $6 / 10$ biopsies the expression correlates positively with the presence of the inhibitor in the primary site. The issue of the presence of membrane bound inhibitors in metastatic tissues, so far was analyzed by Hosch et al. only [17]. In this study, the majority of tumors showed no expression of CD59 in tumor cells. However, this work did not include studies on primary tumors.

In summary, we conclude that further studies are needed to determine the clinical significance of the prevalence of CD46 expression in colon cancer. Possibly the inhibition of this regulator with concurrent immunotherapy could have a beneficial effect on the colon cancer treatment and patients survival.

This study was supported by the State Committee for Scientific Research, grant S3 PO5B 09724.

\section{References}

1. McLaughlin P (2001): Rituximab: perspective on single agent experience, and future directions in combination trials. Crit Rev Oncol Hematol 40: 3-16. 
2. Loberg RD, Day LL, Dunn R, et al. (2006): Inhibition of decay-accelerating factor (CD55) attenuates prostate cancer growth and survival in vivo. Neoplasia 8: 69-78.

3. Watson NF, Durrant LG, Madjd Z, et al. (2006): Expression of the membrane complement regulatory protein CD59 (protectin) is associated with reduced survival in colorectal cancer patients. Cancer Immunol Immunother 55: 973-980.

4. Gelderman KA, Blok VT, Fleuren GJ, Gorter A (2002): The inhibitory effect of CD46, CD55, and CD59 on complement activation after immunotherapeutic treatment of cervical carcinoma cells with monoclonal antibodies or bispecific monoclonal antibodies. Lab Invest 82: 483-493.

5. Bjřrge L, Stoiber H, Dierich MP, Meri S (2006): Minimal residual disease in ovarian cancer as a target for complement-mediated mAb immunotherapy. Scand J Immunol 63: 355-364.

6. Niculescu F, Rus HG, Retegan M, Vlaicu R (1992): Persistent complement activation on tumor cells in breast cancer. Am J Pathol 140: 1039-1043.

7. Alliot C (2003): Edrecolomab in the adjuvant treatment of colorectal carcinoma. Lancet 361: 82; author reply 83.

8. Spizzo G, Obrist P, Went P, et al. (2003): Edrecolomab in the adjuvant treatment of colorectal carcinoma. Lancet 361: 83; author reply 83.

9. Koretz K, Brüderlein S, Henne C, Möller P (1993): Expression of CD59, a complement regulator protein and a second ligand of the CD2 molecule, and CD46 in normal and neoplastic colorectal epithelium. Br J Cancer 68: 926-931.

10. Schmitt CA, Schwaeble W, Wittig BM, et al. (1999): Expression and regulation by interferon-gamma of the membrane-bound complement regulators CD46 (MCP), CD55 (DAF) and CD59 in gastrointestinal tumours. Eur J Cancer 35: 117-124.

11. Thorsteinsson L, O'Dowd GM, Harrington PM, Johnson PM (1998): The complement regulatory proteins CD46 and CD59, but not CD55, are highly expressed by glandular epithelium of human breast and colorectal tumour tissues. APMIS 106: 869-878.

12. Inoue H, Mizuno M, Uesu T, et al. (1994): Distribution of complement regulatory proteins, decay-accelerating factor, CD59/homologous restriction factor 20 and membrane cofactor protein in human colorectal adenoma and cancer. Acta Med Okayama 48: 271-277.

13. Niehans GA, Cherwitz DL, Staley NA, et al. (1996): Human carcinomas variably express the complement inhibitory proteins CD46 (membrane cofactor protein), CD55 (decay-accelerating factor), and CD59 (protectin). Am J Pathol 149: 129-142.

14. Durrant LG, Chapman MA, Buckley DJ, et al. (2003): Enhanced expression of the complement regulatory protein CD55 predicts a poor prognosis in colorectal cancer patients. Cancer Immunol Immunother 52: 638-642.

15. Koretz K, Brüderlein S, Henne C, Möller P (1992): Decay-accelerating factor (DAF, CD55) in normal colorectal mucosa, adenomas and carcinomas. Br J Cancer 66: 810-814.

16. Bjřrge L, Vedeler CA, Ulvestad E, Matre R (1994): Expression and function of CD59 on colonic adenocarcinoma cells. Eur J Immunol 24: 1597-1603.

17. Hosch SB, Scheunemann P, Lüth M, et al. (2001): Expression of 17-1A antigen and complement resistance factors CD55 and CD59 on liver metastasis in colorectal cancer. J Gastrointest Surg 5: 673-679. 